\title{
An Auto-Configuration of 4M Group Management Using Wireless Sensor Networks
}

\author{
Suk-Keun Cha ${ }^{1}$, Jeong-Hoon Lee ${ }^{1}$, Han Gyu Kim², Joon Jae Yoo ${ }^{3}$, Jung Hoon Kang ${ }^{3}$, \\ Dong Hoon Kim ${ }^{4}$, Jun Yeob Song ${ }^{4}$ \\ ${ }^{1}$ Research and Development Center, ACS Co., Ltd., Seoul, Korea \\ ${ }^{2}$ Research and Development Center, SM Information and Communication, Seoul, Korea \\ ${ }^{3}$ Ubiquitous Embedded Fusion Center, Korea Electronics Technology Institute, Seoul, Korea \\ ${ }^{4} U$-Production Team, Korea Institute Machinery and Material, Daejeon, Korea \\ E-mail: \{sk_cha,jh_lee\}@acs.co.kr,hgk@smic.co.kr, \{yoojj, budge\}@keti.re.kr, \{kdh680,sjy658\}@kimm.re.kr \\ Received October 9, 2009; revised November 20, 2009; accepted March 19, 2010
}

\begin{abstract}
The first tier of automotive manufacturers has faced to pressures about move, modify, updating tasks for manufacturing resources in production processes from demand response of production order sequence for motor company and process innovation purpose for productivity. To meet this requirements, it has to require absolutely lead time to re-wiring of physical interface for production equipment, needs for change existing program and test over again. For prepare this constraints, it needs studying an auto-configuration functions that build for both visibility and flexibility based on the 4M (Man, Machine, Material, Method) group management which is supports from Wireless Sensor Network (WSN) of the open embedded device called Machine to Machine (M2M) and major functions of middleware including point manager for real-time device communication, real-time data management, Standard Application Program Interface (API) and application template management. To be application system to Reconfigurable Manufacturing System (RMS) for rapidly response from various orders and model from motor company that is beginning to establishing the mapping of manufacturing resources of $4 \mathrm{M}$ using WSN.
\end{abstract}

Keywords: Auto-Configuration, Wireless Sensor Network, Reconfigurable, Production Resources of 4M, Tinyos, Machien to Machine, Middleware, Application Template Manager

\section{Introduction}

The increasing popularity and applicability of wireless sensor networks (WSNs) in large-scale applications such as environmental monitoring and real-time data integration for manufacturing activities (we called UbiquitousManufacturing) motivates the development of new high-level abstractions for programming.

Typically, a sensor network consists of a large number of nodes deployed in an environment being sensed and controlled. Each node includes one or more sensors, may contain actuators, has limited memory, power and computational capacities.

Wireless sensor networks can be considered distributed computing environments with severe constraints of CPU speed, memory size, power, and bandwidth. Individual nodes in the sensor network are typically unreliable and the network topology may change dynamically.
Sensor networks also differ because of their tight interaction with the physical environment via sensors and actuators. Because of this interaction, sensor networks are very data-centric. Due to all of these differences, many solutions developed for general distributed computing platforms and for adhoc networks cannot be applied to sensor networks. However, sensor networks also exhibit characteristics of both embedded systems and general-purpose systems. They must consume low sensor power and be robust to environmental conditions, while also providing common services that make it easy to write applications [1].

4M Group management service plays a key role in WSNs as it provides support to high level middleware services such as real-time device communication, realtime data management, standard Application Program Interface (API) and application template management, security, fault-tolerance, power management, and so on. 
However, the way a group is managed may vary either from service to service or according to manufacturing activities based on 4M resources (e.g., worker activities, machine running status, error code, tracking of work in process (WIP) and product, manufacturing operation procedures). For example, the group management used by the security service may differ from one used by the fault-tolerance service. In addition, the group management may be reconfigured due to the power level of some nodes in the network.

This paper is organised as follows. Section 2 introduces basic principles of WSNs, 4M group management and reconfiguration. The next section presents our proposal on reconfigurable $4 \mathrm{M}$ group management. Section 4 illustrates how the proposed service may be used to support a particular service. Finally, the last section presents some conclusions and guidelines for further work.

\section{Basic Concept}

This section presents some basic concepts of production resources of $4 \mathrm{M}$, WSNs, group management and reconfiguration in WSNs.

\subsection{Production Resources of the $4 \mathrm{M}$}

Viewpoint of integration for production resources, 4M has consisted of following major categories as referred Figure 4;

- Man

- Machine

- Material

- Method

For data collection from various type of machines, every machine has standalone device controllers as PLC (Programmable Logic Controller), CNC (Computerized Numerical Control), FMS (Flexible Manufacturing System) and robot controller that is automatically interface through standard communication of RS232C or Ethernet with associate protocols by real-time.

For data collection form workers, it must manually report for defects, non-operation reasons and lot numbers that is decision made by workers using a touch screen, bar code reader and mobile devices. It requires tracking of material logistics including Work in Process (WIP) and real-time inventory level from receipt of raw materials to shipping of finished goods.

For data collection of method, bar code reader, IC card, RFID/USN will be able to digitalize for production status information including actual production, quality level, equipment running, optimal scheduling and material logistics that provides all associated production activity tracking for PL (Product Liability) and performance analysis. [2]

The method of digitalization for $4 \mathrm{M}$ defined 3 ways as well as automatic, semi-automatic and manual using wired/wireless multi-sensor data fusion as referred Figure 1.

1) Automatic data collection method: stand alone device controllers has standard communication port such as RS-232C or Ethernet, in this case, associated protocols easy way to automatic data collection by real-time for current status of actual production operation as well as machine running status, actual production report and quality level.

2) Semi-automatic collection method: There are 2 type of method will be available. First, embedded data collection system is cabling with process $\mathrm{I} / \mathrm{O}$ from PLC I/O point or device controllers with $4 \mathrm{M}$ digitalization program. Second, installed additional multi-sensor in device controller or production processes that interface wired/ wireless sensor communication such as RFID/USN.

3) Manual data collection method: workers can directly data entry by touch screen, bar code, RFID and IC card for simple way to operating it in harsh environment.

\subsection{Wireless Sensor Networks}

A wireless sensor network consists of a large number of small devices with computational power, wireless communication and sensing capability [3]. Sensor nodes are usually scattered in an observation region. Each sensor node in the observation region is responsible for extracting data from the environment (such as temperature, humidity, pressure and luminosity), processing and sending them through one or more sink nodes, which are responsible for transmitting these data to the final user.

The design of sensor network applications is highly influenced by resource scarcity (e.g., battery, memory and processor), communication models and application requirements. In wireless transmission, the power of a signal decreases proportionally with the square of the

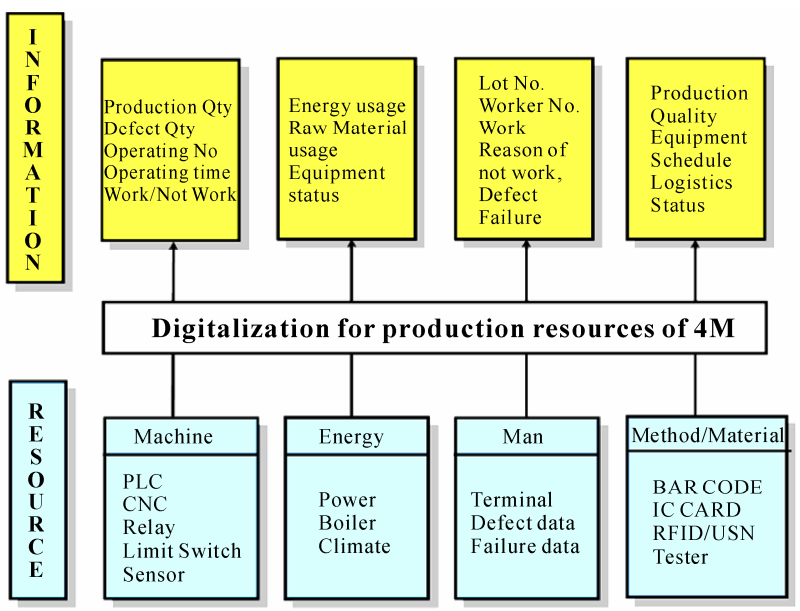

Figure 1. Digitalization for production resources of $4 \mathrm{M}$. 
distance. Thus, if the communication between sensor nodes and the sink will be carried through in a single hop, it will be necessary to adjust signal strength to make possible message exchange in the network. This procedure will cause a power consumption increase and, consequently, a reduction of network lifetime.

One solution would be to adopt a multi-hop communication, where some intermediate nodes act as bridges between the message source and its destination. However, in a dense WSN, various nodes often detect common phenomena leading to unnecessary redundancy of data and transmissions. This fact makes power consumption even more critical. One technique that helps to save energy is data aggregation [4,5]. The idea is to combine the data coming from different sources eliminating the redundancy of the transmitted data, minimizing the number of transmissions and thus saving energy [1]. Application-specific requirements also affect the network's resources in different ways.

For example, the consumption of energy for sending messages to the network tends to be higher in monitoring applications than in surveillance systems since environment violation is a rare event (report by exception). When a violation happens, the application must guarantee the delivery of the event notification in an adequate time. In a tracking application, only the sensors closer to the observed object need to send information to the network.

The application-specific requirements combined with resource restrictions make application development for sensor networks a challenging task. The design of middleware for WSNs must consider these issues in order to hide the complexity from the application developers. However, due to the mission of supporting and optimizing for a broad class of applications, tradeoffs need to be explored between the degree of application-specific and generality of the middleware.

\subsection{Group Management WSNs}

The Group Management service is responsible for the creation and maintenance of groups of sensors/actuators nodes. Basic in this service is the group management model that defines the group structure and how it is managed. Two kinds of group structure are usually adopted: hierarchical or flat. The hierarchical structure has been widely used [6-9] and specifies the existence of a group leader, which is the group representative to interact with other groups and to centralize the group information.

The group leader is also responsible for inter-group communication. In the flat group structure each member knows the other members.

The design of a $4 \mathrm{M}$ group management service for WSNs should follow some principles similar to those applied to Mobile Ad Hoc Networks [10], such as:
1) Resource define: the overhead of group management should be affordable for resource-constrained devices;

2) Distributed: group management cannot accommodate a centralized solution where a single node is always responsible for managing the group. This is due to node mobility and the necessity of load balancing;

3) Dynamism: the group management service must accommodate to the highly dynamic group members of the network. These group members dynamics are caused by network topology changes, power level changes and group management behaviour.

In order to satisfy the aforementioned design principles, the group management service must implement three basic functions: member discovery, group formation and dynamic management.

The member discovery functionality is responsible for discovering the nodes that are eligible for membership according to the group management rules. The group formation task is responsible for identifying the need for a new group. Finally, the group management functionality refers to an ability to update group membership according to the group management rules and network topology changes.

Group Management has been used in sensor networks to support services such as: Fault tolerance (e.g., replication groups), security (e.g., consensus groups), time synchronization (e.g., synchronization groups), object tracking and power management.

The use of a management group is particularly important to power management. It is responsible for minimizing power consumption and making nodes power rest for long durations (from months to years). A promising approach to reduce power consumption in sensor networks is to control the nodes execution. This approach consists of making a small subset of nodes remain active to maintain the network services running while the other nodes sleep or enter in an energy saving mode. This service should be responsible for minimizing energy consumption and maximizing network lifetime. This service can be developed using a Group Management service that is responsible for managing the nodes that remain in the group of nodes responsible for maintaining the network services.

\subsection{Reconfiguration in WSNs}

Reconfiguration and self-adaptation are vital capabilities of sensor networks that are required to operate in dynamic environments that impose varying functional and performance requirements.

Dynamically adaptive software comprises of tasks that detect internal and external changes to the system, reflect on the event occurrences, and adapt to the new conditions. Ad hoc wireless sensor networks, in particular, 
must be designed with adaptation capabilities that enable them to handle a multitude of operating conditions. Reconfiguration in such systems presents significant challenges because of the severe constraints in energy, computation, and communication resources. Runtime technologies that allow software to evolve as system requirements and/or its environment change are critical to the development and deployment of such systems. This is in contrast to the current state-of-the-art in that it does not allow embedded software to evolve at runtime.

\section{4M Group Management Middleware: Auto-Configuration}

A reconfigurable 4M group management service that we called auto-configuration should perform the following operations:

1) Layer structure configuration: it provides an identification of the 4M Group Management services available for reconfiguration;

2) $4 \mathrm{M}$ management: It define $4 \mathrm{M}$ resources and provides $4 \mathrm{M}$ data entry and mapping functions between $4 \mathrm{M}$ resources for verification;

3) Tag Mapping: it returns an identification of the available configurations of a registered tag from $4 \mathrm{M}$ resources with editable function and new registration;

4) 4M Dynamic configuration: it consists of $4 \mathrm{M}$ dynamic mapping the internal behaviour of the Group
Management service currently in use by drag \& drop operation.

The next sections present the design an implementation of the reconfigurable management middleware service-Auto-configuration.

\subsection{Design}

This section presents the design of the proposed reconfigurable group management. The reconfigurable group management is made up of 4 main elements: point manager, real-time data manager, application template manager and auto-configuration (see Figure 2). The RDB stores the group management components available to be used in the reconfiguration process. Meanwhile, the auto-configuration is responsible for the reconfiguration itself. The application asks the auto-configuration to change the group management service to another one existing in the RDB.

The UML diagram shown in Figure $\mathbf{3}$ presents the elements that make up the configurable group management and their relationships. An auto-configuration has pattern structure of the MVC (Model-View-Controller).

MainForm has composites work center for the user interface and control for the internal operation. Each work centers provides 4M Resource Definition, Resource Scheduling, Resource Allocation, Executing \& Data Collection and it can define production type including Batch, Continuous, Discrete for detailed implementation.

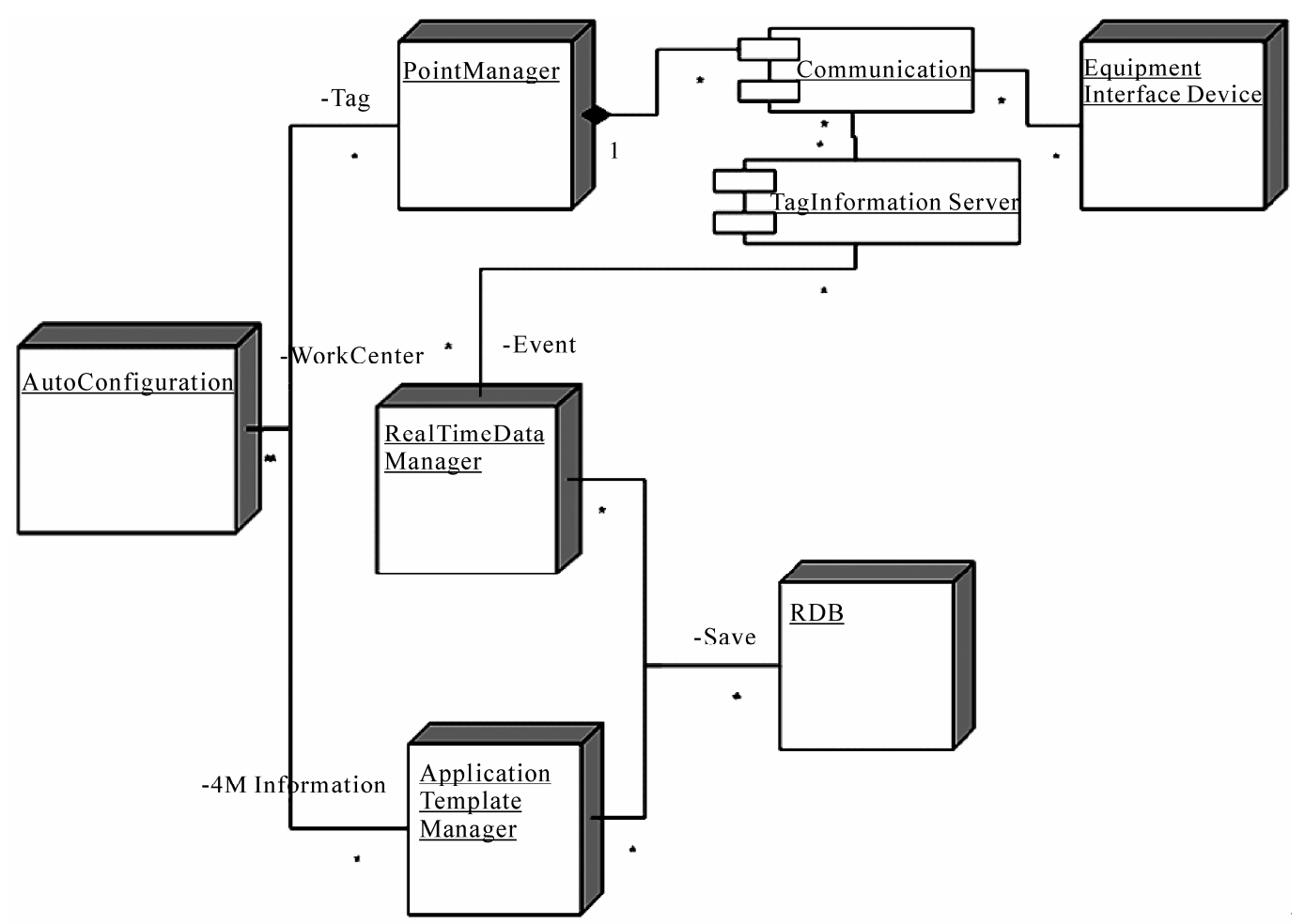

Figure 2. Overview of the auto-configuration. 


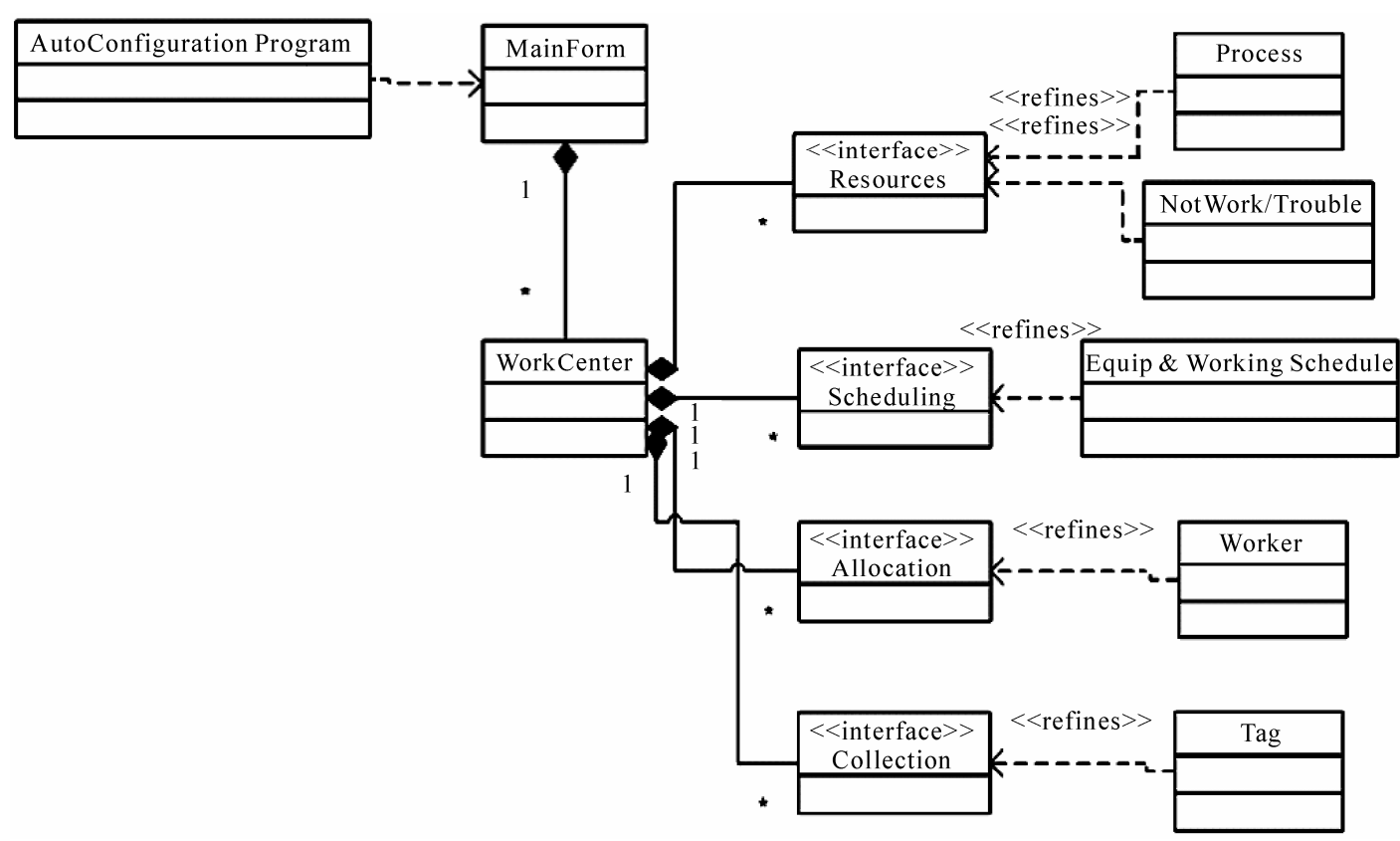

Figure 3. UML diagram of the middleware service.

General Implementation element consisted of process parameters, run/down status data from production equipment and operation plan, etc.

Figure 4 shows operation sequence from Auto-Configuration, it can define resources from activity model based on manufacturing execution management and planning activity model and it runs monitoring step in real-time for the tag definitions to data collection in real-time from the $4 \mathrm{M}$ resources.

Function for execution of the Application Template Manager provides plan, assign and define from 4M resources in the work center. It runs under the mapping by the point manager for data collection from defined tags of the $4 \mathrm{M}$ resources. After this, the $4 \mathrm{M}$ data stores into

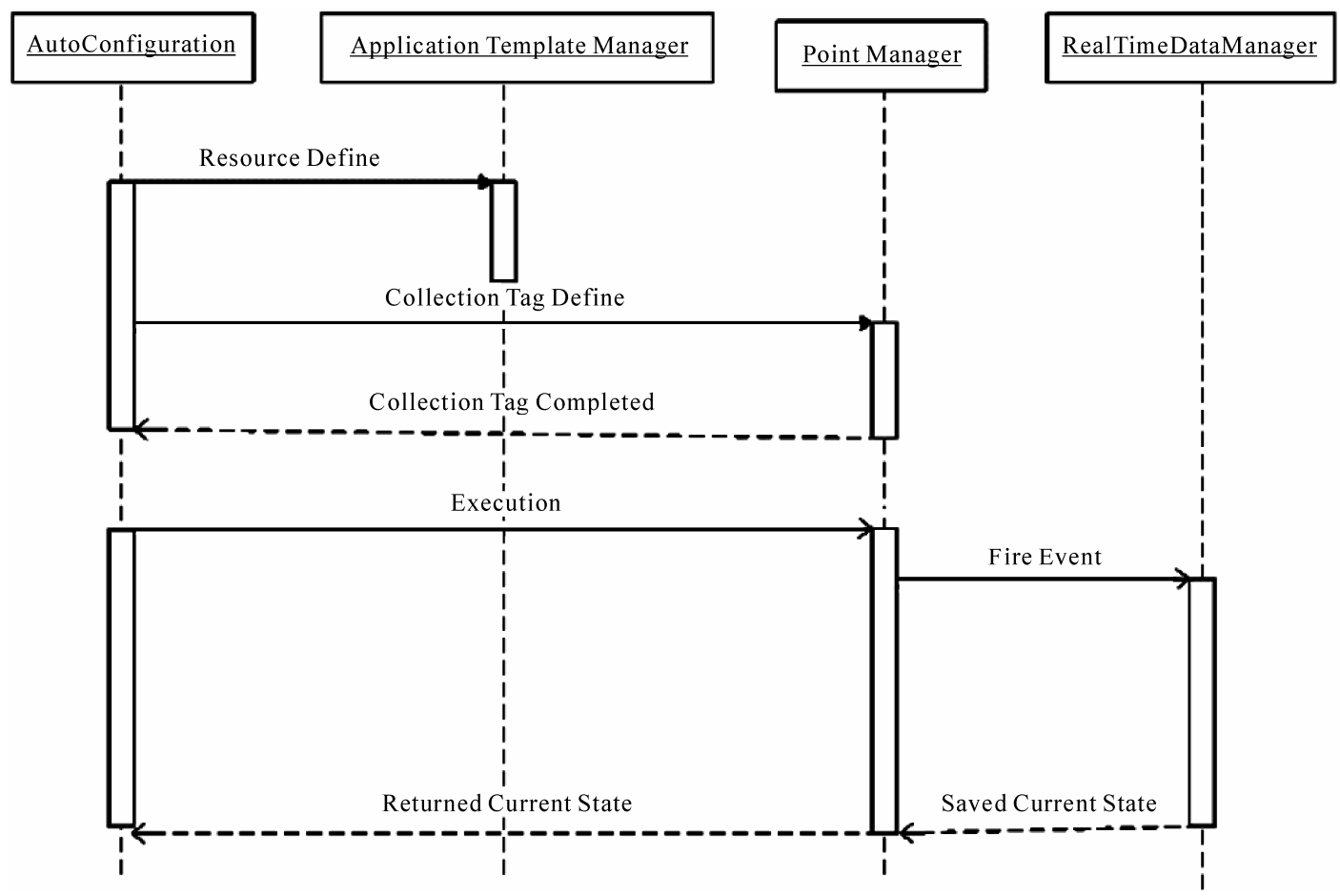

Figure 4. UML diagram of the middleware sequence. 
the real-time data manager.

The UML diagram shown in Figure 3 presents the elements that make up the configurable group management and their relationships. An auto-configuration has pattern structure of the MVC (Model-View-Controller).

MainForm has composites work center for the user interface and control for the internal operation. Each work centers provides 4M Resource Definition, Resource Scheduling, Resource Allocation, Executing \& Data Collection and it can define production type including Batch, Continuous, Discrete for detailed implementation.

\subsection{Implementation}

The u-Manufacturing IT layer consisted of 4 tier layers as following required functions [11];

1) Layer 0: Interface layer provides wired/wireless network through interface with manufacturing resources of $4 \mathrm{M}$ using RFID/USN technology, touch screen and mobile devices. Interface layer must take into consideration high reliable multi-hop function for QoS (Quality of Service), wireless security for 128 bit AES (Advanced Encryption Standard) and how to digitalize 4M for production information.

2) Layer 1: Execution layer provides gateway function runs under middleware such as point manager for common protocol handling of various device controllers, real-time data manager of $4 \mathrm{M}$ data handling by real-time, standard API manager of integration with other application and auto configuration of manufacturing resource of $4 \mathrm{M}$. Functionality of Execution layer provides work-order processing, lot handling, scheduling handling, non-run handling, material handling based 4 zero (It means Zero Inventory, Waiting-time, Defect, Down-time) approach.

3) Layer 2: Management layer provides each plant of ISO Standard MES KPI (Manufacturing Execution System Key Performance Indication) based on managing factors for quality, cost and deliver, which are supported functions, such as quality management, plan \& scheduling management, production execution management, productivity management, inventory management, over equipment efficiency management, production history management, base data management in standard applications that are link data for $4 \mathrm{M}$ in production resources.

4) Layer 3: Planning layer provides standard application function for ERP/SCM/CRM/PDM and so on.

In case of Layer 0 layer of interface, it runs under the Tiny OS with WSN nodes and open embedded Linux device called M2M system for data collection of 4M resources interface by RS232C serial communication, Digital I/O, Analog I/O and Ether- net with protocol drivers such as TCP/IP, MIMOSA (Machinery Information Management Open System Alliance) and OPC (OLE for Process Control) of interoperability standard communication.

Auto-Configuration in the Layer 1 of execution layer with middleware services runs under the Windows NT operating system with .Net framework.

The middleware provide user oriented customization function with WPF (Windows Presentation Foundation) that brings easy to use and faster response for reconfigurable work center in production resources from 4M against with changing various production orders and models.

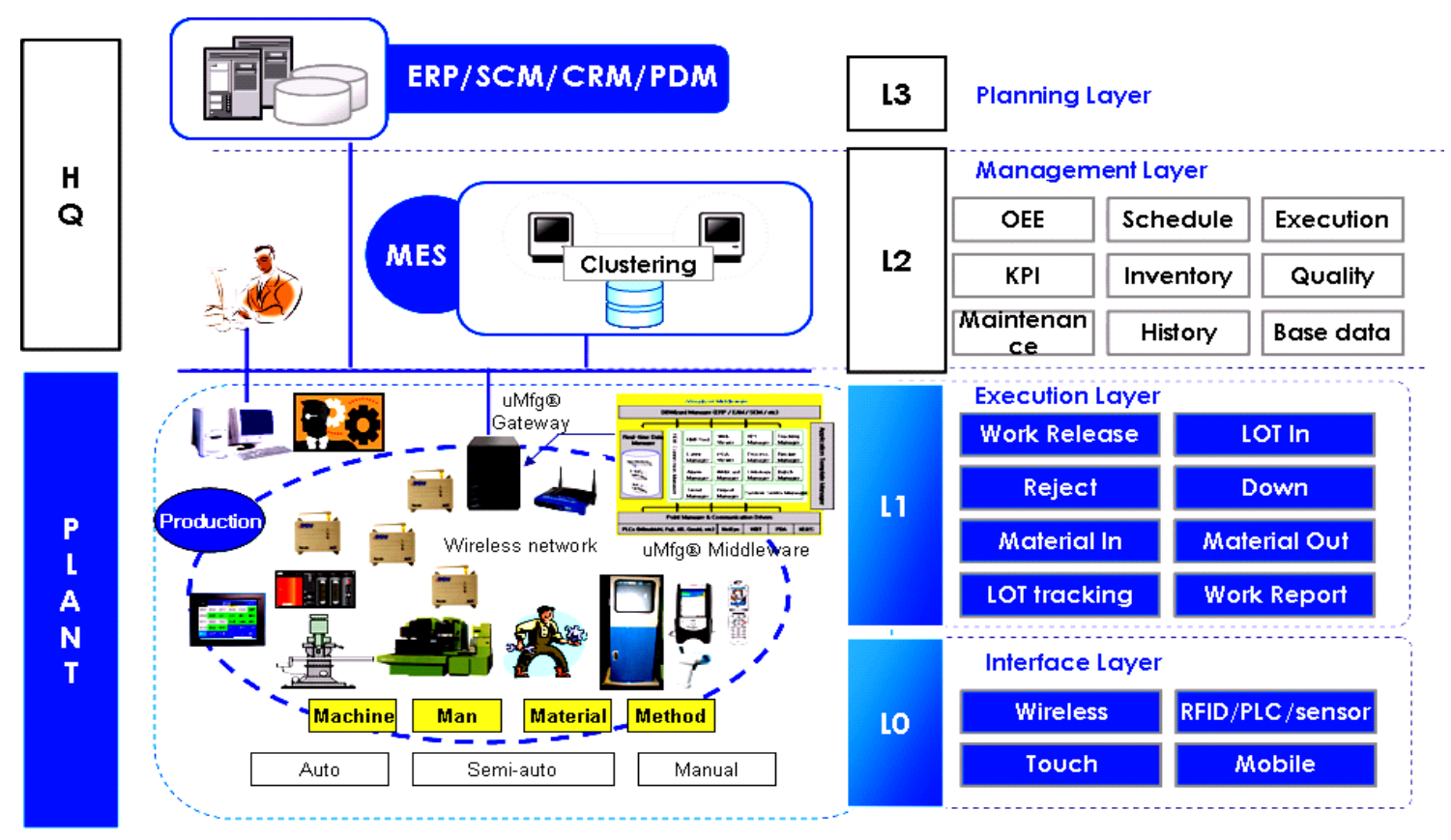

Figure 5. The u-manufacturing IT layers. 


\subsection{Operation of Auto-configuration}

Figure 6 shows user benefits of before and after about operation of the Auto-configuration. Traditional operation environment, it is inevitable to repeat tasks of the redefine for device controller's profile with re-cabling between device controllers and data collection devices and testing with modification of existing control program in servers when it becomes reconfiguration of $4 \mathrm{M}$ resources.

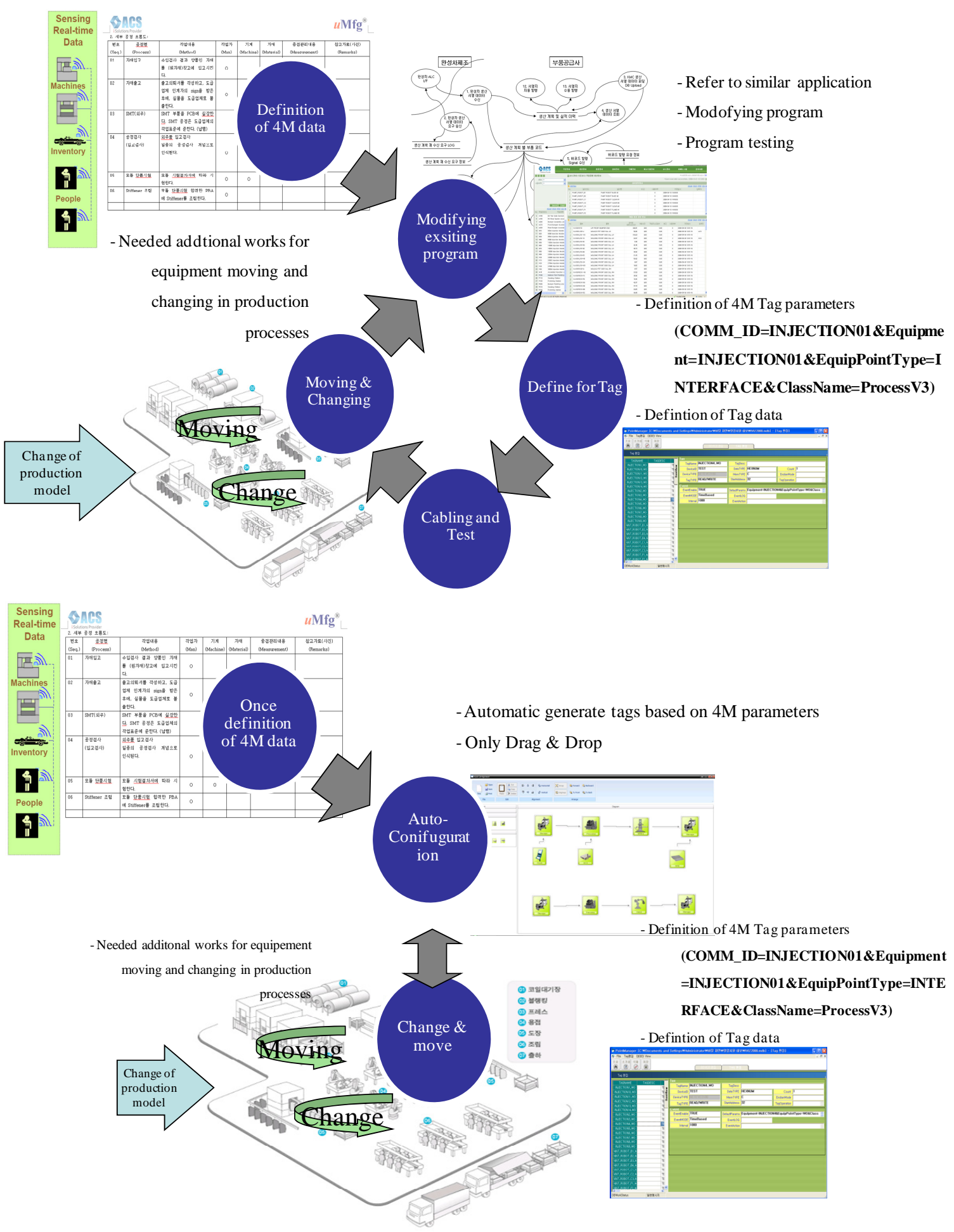

Figure 6. Before and after for using an auto-configuration. 


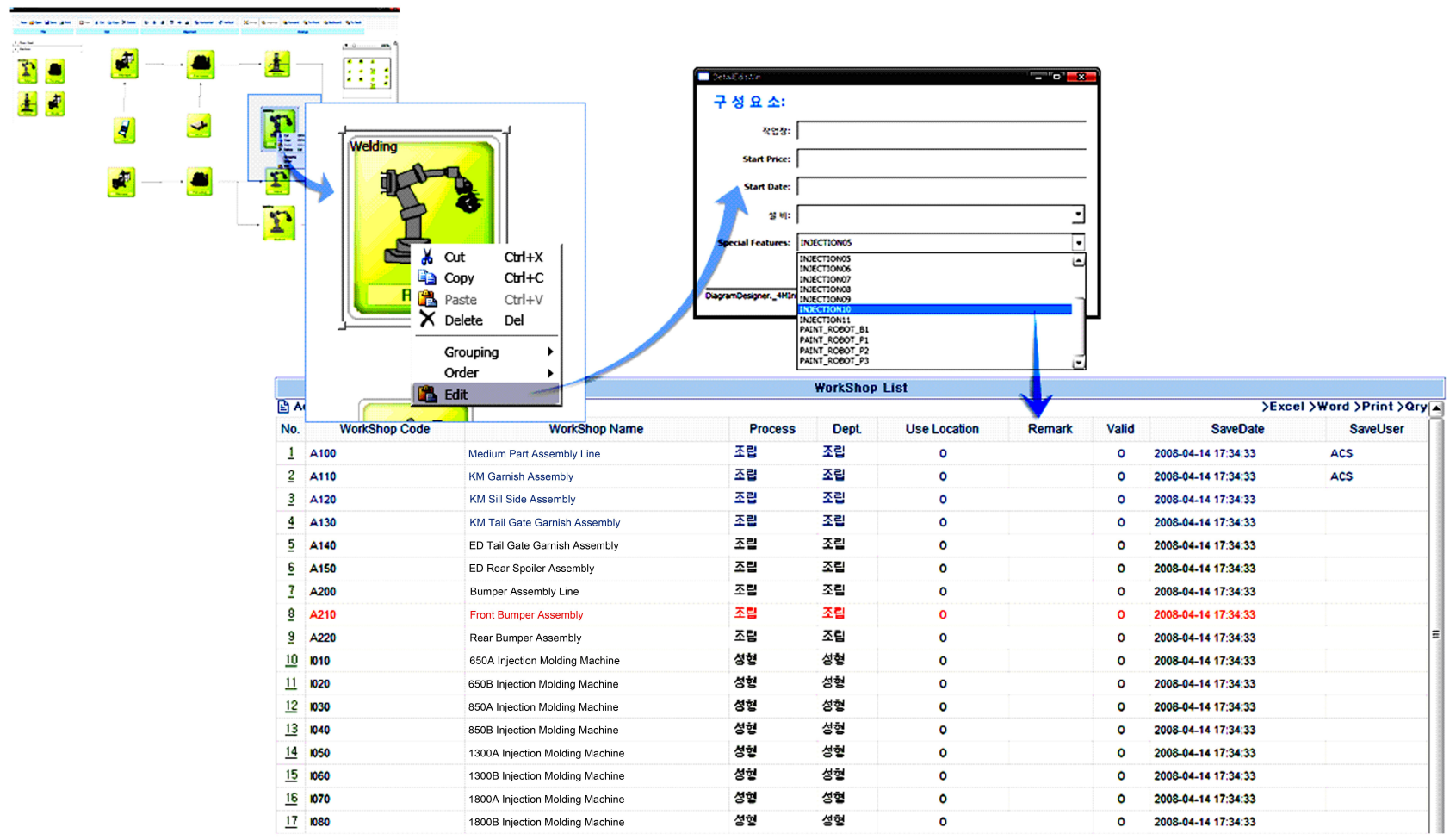

Figure 7. An example for operation procedures of the auto-configuration.

Such a painful tasks from view point of the production technology, it requires knowledge based and know how for fine turning. Major functionality of the Auto-configuration is able to handling above painful repeat work that provides reconfigurable 4M group management.

Users does not need how to define the profile of device controller based on $4 \mathrm{M}$ resources, it needs just drag and drop on the graphic screen of production process if it once define the profile of $4 \mathrm{M}$ resource into the Auto-configuration.

Figure 7 shows simple operation procedures of the Auto-configuration. Base management of the Auto-configuration is work center in production that link to structure of lines, plants. This screen presents how to define the $4 \mathrm{M}$ resources based on work center. Therefore, user does not pay attention to definition of $4 \mathrm{M}$ resources including man, machine, material, method information that has been once defined $4 \mathrm{M}$ resources.

\section{Conclusions}

This paper has presented a reconfigurable group man agement middleware service. The proposed service is designed according to selected guidelines and then implemented in nesC (TinyOS).

The application of the reconfigurable $4 \mathrm{M}$ group management middleware service is illustrated through the implementation of an Auto-configuration application that uses the proposed service. In this particular case, the reconfigurable group management middleware service is used to manage the groups that monitor the $4 \mathrm{M}$ resources being tracked.

Major function of the Auto-configuration for 4M group management with middleware services including point manager, real-time data management, standard API and application template management using wireless sensor network provides RMS for rapidly response from various production orders and models from customers to be establishing manufacturing collaboration.

\section{Acknowledgements}

This work is supported by the Industry Foundation project from the Ministry of Knowledge Economy in the Korean Government.

\section{References}

[1] B. Krishnamachari, D. Estrin and S. B. Wicker, "The Impactof Data Aggregation in Wireless Sensor Networks," Proceedings of the 22nd International Conference on Distributed Computing Systems, Vienna, 2-5 July 2002, pp. 575-578.

[2] SMBA, "Report on E-Manufacturing Project," Korea Institute of Industrial Technology Evaluation and Planning 
(SMBA), Seoul, Vol. 1, June 2004, pp. 34-40.

[3] I. F. Akyildiz, W. Su, Y. Sankarasubramaniam and E. Cayirci, “A Survey on Sensor Networks," IEEE Communications Magazine, Vol. 40, No. 8, August 2002, pp. 102114.

[4] S. Lindsey and C. S. Raghavendra, "PEGASIS: Power Efficient Gathering in Sensor Information Systems," Proceedings of the IEEE Aerospace Conference, Montana, 9-16 March 2002, pp. 1125-1130.

[5] W. Heinzelman, A. Chandrakasan and H. Balakrishnan, "Energy-Efficient Communication Protocol for Wireless Microsensor Networks," Proceedings of the IEEE Hawaii International Conference on System Sciences, Hawaii, Vol. 8, 4-7 January 2000, pp. 8020-8029.

[6] J. Liu, J. Reich, E. Cheung and F. Zhao, "Distributed Group Management in Sensor Networks: Algorithms and Applications to Localization and Tracking,” Telecommunication Systems, Vol. 26, No. 2-4, 2004, pp. 235-251.

[7] J. Liu, J. Reich, E. Cheung and F. Zhao, "Distributed Group Management for Track Initiation and Maintenance in Target Localization Applications," Proceedings of the 2nd International Workshop on Information Processing in Sensor Networks (IPSN'03), Palo Alto, Vol. 2634, 22-23
April 2003, pp. 113-128.

[8] L. Luo, T. F. Abdelzaher, T. He and J. A. Stankovic, "EnviroSuite: An Environmentally Immersive Programming Framework for Sensor Networks,” ACM Transactions on Embedded Computing System (TECS), Vol. 5, No. 3, August 2006, pp. 543-576.

[9] T. Abdelzaher, B. Blum, Q. Cao, Y. Chen, D. Evans, J. George, S. George, L. Gu, T. He, S. Krishnamurthy, L. Luo, S. Son, J. Stankovic, R. Stoleru and A. Wood, "EnviroTrack: Towards an Environmental Computing Paradigm for Distributed Sensor Networks," Proceedings of the 24th International Conference on Distributed Computing Systems, Tokyo, 23-26 March 2004, pp. 582-589.

[10] J. Liu, D. Sacchetti, F. Sailhan and V. Issarny, "Group Management for Mobile Ad Hoc Networks: Design, Implementation and Experiment," Proceedings of the 6th International Conference on Mobile Data Management (MEM’05), Ayia Napa, 9-13 May 2005, pp. 192-199.

[11] S.-K. Cha, J.-S. Choi, C.-S. Choi and W.-S. Kim, "Counter Plan and Strategy for Small \& Medium Enterprises for Ubiquitous Age,” KIMI, December 2004, pp. 63-171. 